\title{
De los juegos a la gamificación: propuesta de un modelo integrado
}

\section{Laura Marcela Londoño Vásquez}

https://orcid.org/oooo-0002-5619-3935 Universidad de Antioquia, Colombia laura.londonog@udea.edu.co

\section{Miguel David Rojas López}

https://orcid.org/0000-0002-3531-4910 Universidad Nacional de Colombia, Colombia mdrojas@unal.edu.co

\section{Resumen}

El interés por temas relacionados con juegos dentro de los procesos educativos se remonta a numerosas propuestas que surgen a partir de los años 60 y a su aplicabilidad en diferentes disciplinas: militar, salud, ciencias sociales e ingeniería, etc. Este documento muestra la evolución desde la forma básica del juego hasta conceptos recientes, como juegos serios y gamificación. Presenta una revisión teórica de diferentes estudios realizados en el campo de juegos en la educación, sus caracteristicas y aplicaciones. En un segundo momento, el artículo propone un modelo teórico integrador de los conceptos asociados a los juegos en la educación, que explica las relaciones entre aprendizaje y diversión.

\section{Palabras clave (Fuente: tesauro de la Unesco)}

Aprendizaje activo; didáctica escolar; gamificación; juegos educativos; método de enseñanza; técnicas de enseñanza.

Recepción: 25/11/2019 | Envío a pares: 05/03/2020 | Aceptación por pares: 23/03/2020 | Aprobación: 19/06/2020 


\title{
From Games to Gamification: A Proposal for an Integrated Model
}

\begin{abstract}
The interest in game-related topics within educational processes is due to numerous proposals that emerged in the 1960 s and their applicability to different disciplines, such as military, health, social sciences, and engineering. This paper shows the evolution from the primary form of the game to current concepts such as serious games and gamification. It presents a theoretical review of various studies conducted in the field of gaming in education, its characteristics, and its applications. Then, it proposes an integrating theoretical model of the concepts associated with gaming in education, which account for the learning-fun relationships.
\end{abstract}

\section{Keywords (Source: Unesco thesaurus)}

Activity learning; school didactics; gamification; educational games; teaching methods; teaching technics. 


\section{Dos jogos à gamificação: proposta de um modelo integrado}

Resumo

O interesse por temas relacionados a jogos dentro dos processos educativos deve-se a numerosas propostas que surgem a partir dos anos 60 e a sua aplicabilidade em diferentes disciplinas, como militar, saúde, ciências sociais e engenharia. Este documento mostra a evolução desde a forma básica do jogo até conceitos recentes, como jogos sérios e gamificação. Apresenta uma revisão teórica de diferentes estudos realizados no campo de jogos na educação, suas características e aplicações. Num segundo momento, o artigo propõe um modelo teórico integrador dos conceitos associados aos jogos na educação, que explica as relações entre aprendizagem e diversão.

\section{Palavras-chave (Fonte: tesauro da Unesco)}

Aprendizado ativo; didática escolar; gamificação; jogos educativos; método de ensino; técnicas de ensino. 
Según Medrano (2005), los seres humanos se divierten con los juegos de mesa desde tiempos remotos y fueron los romanos los que inventaron la palabra Ludo, que significa jugar; por esta razón todo lo relacionado con el juego se denomina lúdico. Una de las definiciones más importantes del término juego fue propuesta por Huizinga (1949), según el cual el juego es "una acción u ocupación libre, que se desarrolla dentro de unos límites temporales y espaciales determinados, según reglas obligatorias, aunque libremente aceptadas". Él propone que esta definición permite comprender lo que se denomina juego en diferentes ámbitos, según se trate de animales, niños o adultos.

Los juegos son actividades que acompañan a las personas desde temprana edad, debido a que por medio de ellos se estimula a los niños para el desarrollo de nuevas estructuras mentales. En las primeras etapas, el juego ayuda a ejercitar y desarrollar esquemas motores; en una segunda etapa, ayuda a la imaginación y consolida la posibilidad de ficción, y en la tercera, el niño acepta reglas que comparte (Bernabeu y Goldstein, 2012). Así tiene un papel fundamental en el desarrollo de habilidades motrices y cognitivas propias de cada edad. En los últimos años, los juegos se convierten en algo más que en actividades recreativas, integrándose al campo del conocimiento y transformándose en herramientas para el aprendizaje en diferentes contextos educativos y organizacionales.

Las definiciones no necesariamente describen el mismo aspecto de los juegos: unas se centran en el juego y otras en la actividad de jugar un juego (Juul, 2010). Por lo anterior, Teixes (2014) plantea que es necesario diferenciar los juegos libres de los niños, los cuales son actividades lúdicas, sin objetivos, que surgen de sus propias motivaciones y en total libertad, los cuales se asocian con el concepto de play (jugar), de los juegos con normas y objetivos concretos que, por su parte, se relacionan con el concepto de game (juego). Gray et al. (2012) los definen como actividades que tiene componentes básicos que los separan de otros tipos de actividades: espacio de juego, límites, reglas de integración, artefactos y meta. Por su parte, Salen y Zimmerman (2004) los definen como un sistema en el cual los participantes se involucran en un conflicto artificial definido por reglas, que arroja un resultado cuantificable.

Así, cuando se hace referencia al juego, no solo se mencionan los juegos destinados al entretenimiento, generalmente con propósito comercial y en los cuales el aprendizaje no es la meta principal, ya que, por otro lado, hay juegos destinados específicamente al aprendizaje (Braghirolli et al., 2016).

De esta manera, los juegos han evolucionado y toman un papel relevante: dentro del contexto educativo, debido al uso en ambientes de educación formal e informal,y dentro de las organizaciones, en el entrenamiento empresarial y la enseñanza organizacional. Es así como en 1970 se empieza a usar el término "juegos serios" para hacer referencia a los juegos diseñados y aplicados principalmente con un propósito educativo,y posteriormente, en el 2010, aparece la "gamificación" como herramienta emergente que usa elementos y diseños propios de los juegos en contextos no lúdicos.

Los juegos en contextos educativos son utilizados en diversas disciplinas, como ciencia, tecnología, ingeniería, matemáticas, salud, negocios, lenguaje, economía, entre otras, e incluso en contextos de aprendizaje extracurricular, como en educación sobre el abuso de sustancias y alimentación saludable (Boyle et al., 2016), lo que demuestra su versatilidad y aplicabilidad en variados contextos educativos y sociales y en distintos niveles de formación. La ingeniería constituye un área en la cual los juegos han tenido importancia e influencia, principalmente en las ciencias de la computación, pero también en procesos de enseñanza de diferentes sujetos de la ingeniería.

El objetivo fundamental de esta investigación es mostrar la evolución que han tenido los juegos en 
la historia reciente y su aplicación y relevancia en los contextos educativos, por medio de diferentes términos y herramientas que surgieron como juegos serios y gamificación, y explicar las relaciones existentes entre dichas herramientas. Se quiere mostrar sus principales definiciones, características y aplicaciones, así como su alto potencial en los procesos de enseñanza-aprendizaje. Para esto se realizó una revisión bibliográfica que muestra la evolución de las herramientas y permite determinar el estado actual de las publicaciones científicas relacionadas con juegos en contextos educativos. Posteriormente, se propone un modelo teórico integrador de los conceptos asociados a los juegos en la educación (juegos, aprendizaje basado en juegos, juegos serios y gamificación), el cual muestra y explica las relaciones existentes a partir del aprendizaje y la diversión.

La estructura del artículo tiene cuatro secciones: la primera corresponde a la introducción y conceptualización general de los juegos; la siguiente hace referencia a los juegos dentro de procesos educativos y muestra su valor e importancia dentro del aprendizaje, para, posteriormente, profundizar en juegos serios y gamificación; a continuación se muestran los principales resultados del análisis y revisión de diferentes estudios y el modelo teórico integrador propuesto.

\section{Juegos en la educación}

Aunque existen variedad de elementos presentes en los juegos, cuando se habla de juegos en la educación se definen como la actividad en la cual los jugadores participan en un contexto determinado, a partir de la descripción general de una situación o sistema delimitado, sometido a reglas, límites u obligaciones, todo con el objetivo de obtener un resultado o desempeño específico al final de la actividad y dar retroalimentación. Gray et al. (2012) proponen que cada juego es un mundo que evoluciona en etapas, que pasan por imaginar y crear dicho mundo, para abrirlo luego, de modo que el participante pueda explorarlo y, finalmente, cerrarlo.
Sin embargo, de acuerdo con Braghirolli et al. (2016), la literatura usa varios términos para describir y hacer referencia a los juegos en contextos educativos (Educational Games), como son:

- Juegos digitales para el aprendizaje (digital learning games).

- Aprendizaje basado en juegos (game-based learning).

- Juegos de entretenimiento educativo (edutainment games; Breuer y Bente, 2010).

- Juegos persuasivos (persuasive games).

- juegos epistémicos (epistemic games).

- Juegos de instrucción (instructional games).

- Juegos serios (serious games; Connolly et al., 2012).

A pesar de las diferentes designaciones dadas a los juegos en contextos educativos, como anotan Braghirolli et al. (2016), las discusiones en cada uno de los estudios contribuyen a entender la utilidad de los juegos en la educación. Es importante mencionar que en muchas ocasiones dichos términos son utilizados como sinónimos, debido a que no existe una clasificación general de los juegos, aunque todos tienen un propósito educativo.

Braghirolli et al. (2016), además, exponen que las simulaciones son el género más común en los juegos educativos, los cuales tienen la habilidad de representar situaciones de la vida cotidiana y permitir a los participantes actuar como administradores, pilotos, físicos, entre otros, en un contexto de juego. Sin embargo, sin los elementos propios de los juegos dentro de la educación, solo se tendrían simulaciones para el entrenamiento. Investigadores han demostrado la efectividad de las versiones para el aprendizaje de juegos existentes en diferentes temas. De esta manera, modificar un juego existente y hacerlo adecuado para soportar actividades educativas es un procedimiento común (Khenissi et al., 2015). 
Los participantes en juegos educativos durante su desarrollo deben entender el problema presentado, idear un plan y ponerlo a prueba para resolverlo, es decir, deben analizar las estrategias para resolver el problema ellos mismos, generar soluciones creativas y de esta manera lograr aprendizaje efectivo (Liu et al., 2011). El participante experimenta por sí mismo, resuelve problemas y situaciones, interactúa con diferentes variables y desempeña roles, desde perspectivas que, de otro modo, sería imposible llevar a cabo en la vida real (Ouariachi et al., 2017), es decir, crea un ambiente seguro para la experimentación, en la cual las consecuencias no son transferidas al mundo real (Braghirolli et al., 2016).

Waiyakoon et al. (2015) plantean que el aprendizaje basado en juegos es una instrucción que utiliza medios de comunicación para el aprendizaje. Los participantes ganarán conocimiento por medio del juego, que los ayudará a crear conocimiento propio en su nivel de retención y comprensión, además que motiva a los participantes a asimilar e interactuar en el proceso de aprendizaje, hasta que logran aprender por ellos mismos.

Los juegos tienen características interactivas y son capaces de revelar los efectos de acciones particulares, lo que permite establecer y probar nuevas estrategias (Braghirolli et al., 2016) y les ofrece a los estudiantes la oportunidad de aprender haciendo, involucrándolos en una experiencia simulada del mundo real, con lo cual se logra una inmersión en una auténtica situación organizacional (Ben-Zvi, 2010), se realza la experiencia y se acumula memorias, conduciendo a respuestas ágiles y a una acelerada capacidad de reacción en situaciones diarias (Lauche et al., 2009). Esto ayuda a que los estudiantes logren habilidades técnicas y una perspectiva de gestión de problemas.

Una característica importante es su versatilidad, dado que permiten lograr diferentes objetivos con los participantes, como es diagnosticar y mejorar procesos organizacionales, enseñar o reforzar conceptos de una temática específica, determinar situaciones de comportamiento individual y grupal, por medio de actividades que hacen del aprendizaje un proceso amigable y a su vez permiten evaluar habilidades en los individuos y grupos (Londoño, 2014).

El aprendizaje es el resultado esperado de un juego educativo (Braghirolli et al., 2016). Como indican Connolly et al. (2012) y Boyle et al. (2011), las teorías modernas del aprendizaje efectivo sugieren que este es más efectivo cuando es activo, experiencial, situado, basado en problemas y proporciona información de forma inmediata, condiciones que se pueden logran por medio de juegos. Adicionalmente, según Iten y Petko (2016), para lograr un efecto óptimo de aprendizaje, la diversión del juego debe estar estrechamente relacionada con el proceso de aprendizaje. Juego y aprendizaje deben conectarse integralmente, en lugar de simplemente alternarse durante el desarrollo del juego. La clave para el éxito de los juegos es encontrar el balance entre diversión y aprendizaje (Moreno-Ger et al., 2008).

Su creciente uso en ambientes de enseñanza y los esfuerzos para mejorar la educación formal con los juegos son influenciados en gran medida por el contexto particular de aprendizaje en el que un juego se despliega y especialmente por la manera como el educador adopta un juego para hacer frente a los objetivos particulares y las metas de aprendizaje (Arnab et al., 2012).

Como exponen Sauvé et al. (2009), algunos estudios demuestran que los juegos establecen condiciones favorables para el aprendizaje, incluyendo la retroalimentación, interacción y participación activa de los participantes. De esta manera la aplicación de juegos está cada vez más vinculada con el aprendizaje y se observan distintos resultados (Connolly et al., 2012):

- Resultados cognitivos: declarativo, procedimental y de conocimiento estratégico.

- Resultados afectivos: creencias o actitudes. 
Los juegos educativos toman una posición relevante como herramientas de apoyo en los procesos de aprendizaje, no solo por la versatilidad y motivación que logran en los participantes, sino por la posibilidad de incluirlos en la enseñanza en diversas áreas del conocimiento y lograr un aprendizaje efectivo por medio de práctica y experimentación. Por ello los diseñadores de juegos trabajan con los profesores para desarrollar e incorporar elementos de juegos en las escuelas (Lee y Hammer, 2011) y centros de aprendizaje.

De esta manera, como herramientas de apoyo en la educación, los juegos constituyen un campo de estudio creciente, que en los últimos años se desarrolla de manera activa, pero que existe hace varias décadas y cada día adquiere mayor relevancia en los procesos de enseñanza-aprendizaje dentro de los centros educativos básicos y universitarios, así como en organizaciones, debido a la versatilidad en la aplicación, el impacto en los participantes y la posibilidad de propiciar el aprendizaje activo; sin embargo, es un área de estudio que aún tiene numerosos e importantes retos dentro de la educación formal, en los procesos de diseño de herramientas y juegos, en la aplicación, recolección y análisis de la información resultante y en la verificación los resultados de aprendizaje.

\section{Juegos serios (serious games)}

El término juegos serios (serious games) es una denominación que toma más fuerza dentro de los juegos enfocados en los procesos de enseñanzaaprendizaje. La adopción inicial del término se atribuye a Clark C. Abt en el libro Serious games (1970), donde los definió, señalando que estos juegos tienen un propósito educativo explícito y cuidadosamente pensado y no están destinados a ser jugados principalmente por diversión, lo cual no significa que los juegos serios no puedan ser entretenidos, definición que según Djaouti et al. (2011) se ajusta a su uso en la actualidad.
Abt es un investigador que trabajó en un laboratorio de los Estados Unidos durante la Guerra Fría, en el cual una de las metas era el uso de juegos para el entrenamiento y la educación (Djaouti et al., 2011). Este tipo de juegos son definidos como "serios" debido a que el término hace referencia a juegos que hacen énfasis en el aprendizaje (Braghirolli et al., 2016). Y aunque según Huizinga (1949) "en nuestra conciencia el juego se opone a lo serio", debido a que se asocia con la risa, el autor manifiesta que el juego puede ser algo muy serio.

La definición inicial de juegos serios hace referencia a juegos que no necesariamente se basan en un soporte digital, pensados con un propósito educativo. Como aseguran Michael y Chen (2006), son juegos en los cuales la educación, es sus diversas formas, es el objetivo principal, más allá del entretenimiento. Son juegos de diversos tipos que difieren en diseño y complejidad y mejoran un conjunto de resultados de aprendizaje diferentes (Riemer y Schrader, 2015).

Otros autores retoman esta definición y van más allá, al relacionar los juegos serios con el uso de herramientas computarizadas y los definen como cualquier uso significativo de los recursos de los juegos computarizados/industria del juego, cuya misión principal no es el entretenimiento (Sawyer, 2007). Por su parte, Khenissi et al. (2015) proponen que son juegos digitales que educan, entrenan e informan. Estos juegos son diseñados con un propósito principal diferente al entretenimiento, el disfrute o la diversión. La intención inicial de los juegos serios es combinar los aspectos serios (aprendizaje, instrucciones, etc.) con los aspectos del juego digital. Desde la perspectiva de Iten y Petko (2016) es común que los juegos hagan que el aprendizaje sea divertido, aunque no es clara la relación de la diversión en los juegos serios y cómo se relaciona con el compromiso cognitivo.

La aplicación de dichos juegos es diversa y comprende una amplia variedad de temas. Según Zyda 
(2005), los juegos serios utilizan el entretenimiento para el entrenamiento en gobierno o corporaciones, educación, salud, política pública y objetivos estratégicos de comunicación. Incluso, según Djaouti et al. (2011), muchos juegos fueron diseñados con objetivos serios antes del 2002 y en diversas áreas del saber, como se muestra en la Figura 1.

Adicionalmente, Djaouti et al. (2011) hacen referencia a que existe una "ola de juegos serios" que comienza alrededor del 2002, año en que se produjo el lanzamiento del juego America's Army, considerado como "el primer juego serio exitoso y bien ejecutado que ganó la conciencia pública total", el cual logra poner el término juegos serios en la mente de muchas personas a partir del 2002.

La aparición del movimiento de los juegos serios se centró en los juegos diseñados para enseñar contenido académico y habilidades a los estudiantes (Kafai y Burke, 2015). Actualmente, la mayoría de los juegos serios tienden a seguir la línea de los juegos digitales, en lugar de seguir la definición más amplia de juegos serios, propuesta para ambos, digitales y no digitales, introducida en los años 70 por Abt (Djaouti et al., 2011). En adición, la atención también se ha centrado en utilizar juegos serios en el entrenamiento en contextos informales (Arnab et al., 2012), en áreas de aplicación por fuera de la educación y el aprendizaje, como arte, terapia, publicidad, entre otras (Breuer y Bente, 2010).

La rápida expansión de los juegos serios en contextos educativos formales e informales se debe a la existencia de por lo menos seis propiedades claves, que señalan las investigaciones, para promover los procesos de aprendizaje (Ahrens, 2015):

1. Un sistema de reglas y una meta a la cual el jugador está unido emocionalmente.

2. Experiencias que ofrecen buenas oportunidades de aprendizaje.

3. Nivelación del objetivo determinante para el presente y la efectividad.

4. Modelamiento, para hacer del aprendizaje una experiencia más general y abstracta.

\section{Figura 1. Distribución del mercado de juegos serios realizados antes de 2002 [953 juegos]}

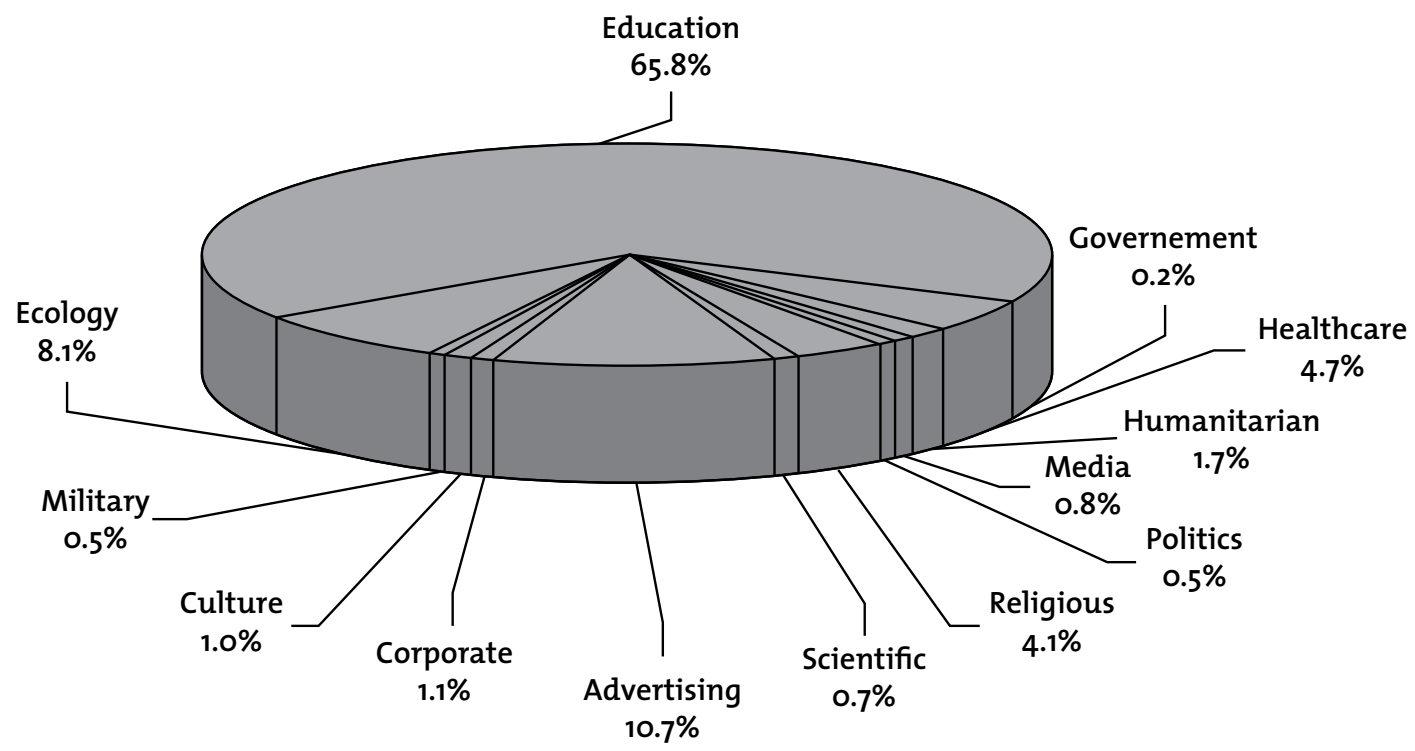

Fuente: Djaouti et al. (2011) 
5. Motivación a los jugadores a definir su propiay única trayectoria a través del juego.

6. Interfaz fácil y amigable con el usuario.

Uno de los riesgos intrínsecos más importantes de los juegos serios es la tentación de priorizar el objetivo serio que tiene el diseñador, sobre la experiencia de los jugadores (Ferrara, 2013), lo que haría que el juego no sea lo suficientemente atractivo y motivador para los participantes. Por esta razón, son numerosos los retos que tiene un diseñador de juegos para lograr el aprendizaje de los participantes; sin embargo, cada día son más las investigaciones y aplicaciones que se realizan sobre el tema, las cuales permiten también la construcción de un marco general que guíe el uso de los juegos serios en el aprendizaje.

De la misma manera, los juegos serios son utilizados dentro de las organizaciones como herramientas de enseñanza, entrenamiento y apoyo para la toma de decisiones. Ahrens (2015) propone que existen cuatro requerimientos fundamentales para su uso en contextos organizacionales:

1. Mostrar la realidad empresarial lo más cerca posible, teniendo en cuenta la organización, productos y procesos.

2. Funcionar con datos corporativos reales, para aproximarse lo más posible a la realidad.

3. Hacer fuertes exigencias en materia de seguridad, relacionado con el acceso no autorizado.

4. Ser fácilmente adaptables.

Como se ha mostrado anteriormente, la incursión de los juegos dentro de los contextos educativos se ha dado de manera acelerada desde el año 2002, principalmente con el desarrollo de los llamados juegos serios. Ello evidencia la importancia y creciente aplicación de juegos en ámbitos educativos, formales e informales, y en ámbitos empresarial, donde es importante que los participantes, por medio de la combinación de contenido y herramientas digitales, logren objetivos específicos de aprendizaje. Los juegos serios son un medio para la construcción activa, en lugar de la recepción pasiva de conocimiento (Arnab et al., 2012).

\section{Gamificación}

Los juegos se encuentran en todas partes. Las personas juegan mientras viajan, descansan e incluso en el trabajo, cuando las organizaciones motivan a sus empleados o clientes con juegos e incentivos, para crear experiencias divertidas (Robson et al., 2015). Debido al creciente uso de juegos en diferentes contextos, tanto formarles como informales, estos y sus elementos han evolucionado en un fenómeno llamado gamification -que podría traducirse por ludificación o gamificación, que es como se lo conoce en español-, que ha ido ganando recientemente atención científica (Groh, 2012).

La gamificación es un término que se origina en la industria de medios digitales. El primer uso documentado data de 2008, aunque es a finales de 2010 cuando el término es adoptado de manera general (Deterding et al., 2011) y empezó a atraer la atención generalizada de los juegos en diversos contextos (Robson et al., 2015), aunque existía y era aplicada por las organizaciones desde antes. Es un término inventado para describir la aplicación de la mecánica del juego, como puntos, insignias y niveles, a procesos que no son juegos (Smith-Robbins, 2011). Por su parte, Deterding et al. (2011) definen la gamificación como el uso de elementos del diseño de juegos, en contextos que no son juegos. Esto la diferencia de los juegos serios y de los diseños para interacciones lúdicas.

Tiene diversas aplicaciones dentro de diferentes contextos. Según Robson et al. (2015), ella utiliza lecciones del dominio del juego para lograr cambios de comportamiento, en situaciones que no son de juego. Por su parte, Gartner (2015, citado por Emel'yanenko et al., 2016) señala que la gamifi- 
cación involucra usuarios y consumidores y mejora su participación en la resolución de problemas aplicados, razones por las que su aplicación se hizo popular dentro de los contextos de mercadeo y ventas.

Por otro lado, dentro del contexto formativo, la gamificación evoluciona como una técnica popular para mejorar los resultados en la educación y en el aprendizaje organizacional (Landers, 2014). Kapp (2012) la define como la aplicación cuidadosa y considerada de los principios del juego para resolver problemas y fomentar el aprendizaje, utilizando elementos apropiados del juego. Esta puede cambiar el comportamiento de los participantes, porque aprovecha las principales motivaciones del comportamiento humano de dos maneras conectadas entre sí: refuerzos y emociones (Robson et al., 2015), con lo que se obtiene un aprendizaje efectivo y con motivación.

De esta manera es posible observar que la gamificación aplica elementos y conceptos propios de los juegos, para lograr comportamientos deseados, resolver problemas o fomentar el aprendizaje. Es la entrega de contenido, con un propósito diferente al entretenimiento puro, usando pensamiento basado en juegos y mecanismos (Kapp, 2012).

Dentro de los contextos educativos, dicha técnica ha sido ampliamente utilizada, ya que, según Kapp (2012), por medio de ella se pueden alcanzar varios tipos de conocimiento, como son: declarativo, conceptual, reglamentado, procedimental, de habilidades blandas, afectivo y de dominio psicomotor, lo que demuestra su versatilidad y utilidad a la hora de incluirla en ambientes de aprendizaje. Adicionalmente, Lee y Hammer (2011) y Emel'yanenko et al. (2016) plantean que la gamificación puede ser usada en numerosos procesos en la educación, debido a que:

- Mejora la motivación de los estudiantes para adquirir nuevo conocimiento, como pensamiento crítico y habilidades para la toma de decisiones.

- Revela el potencial creativo de los participantes.
- Es un medio para mejorar la educación vocacional en las universidades.

- Proporciona a los profesores mejores herramientas para guiar y recompensar a los estudiantes.

- Hace que los estudiantes vayan en la búsqueda del conocimiento, inspirándolos a aprender a lo largo de toda su vida.

- Muestra la manera en que la educación puede ser una experiencia alegre y divertida.

Se trata de motivación y compromiso. Hacer el aprendizaje divertido no requiere altas inversiones en tecnología. En su lugar, enfocando las maneras en que la tecnología del entretenimiento nos compromete, puede aportar métodos susceptibles de ser transferidos a cualquier situación de aprendizaje (Smith-Robbins, 2011). De esta manera, la gamificación se convierte en una herramienta poderosa que, al incluirla en los procesos de enseñanza-aprendizaje, potencia la motivación, ayuda en la adquisición de conocimiento, así como en la inmersión de los participantes en ambientes de enseñanza. Adicionalmente, en la investigación realizada por Hamari et al. (2014) se señala que, aunque la gamificación puede no ser a largo plazo, sus resultado podrían ser resultado de un efecto de novedad.

En las organizaciones, la gamificación es una manera de reforzar el servicio por medio de un sistema basado en reglas que proporcionan al usuario información, retroalimentación y mecanismos de interacción, con el objetivo de facilitar y apoyar la creación de valor general para los usuarios (Huotari y Hamari, 2011).

De esta manera, tiene gran potencial de aplicación en contextos del cuidado de la salud, sostenibilidad, gobierno, transporte, educación (Robson et al., 2015), gestión de personal, innovación, mercadeo, planeación de finanzas personales (Emel'yanenko et al., 2016), entre otros. Es así como grandes compañías de bebidas, telecomunicaciones, energía y 
automotores ya usan programas de fidelización en línea, con puntos, niveles o insignias, que permiten atraer a los usuarios o consumidores y mantener las relaciones (Robson et al., 2015).

Los proyectos de gamificación deben ser cuidadosamente diseñados, para enfocarse en los retos y objetivos. Ello porque, según Gartner (2011, citado en Robson et al., 2015), aproximadamente el $80 \%$ de las aplicaciones gamificadas podrían fallar en el logro de los objetivos, principalmente debido a que los procesos han sido gamificados de manera inapropiada o también por falta de comprensión de lo que realmente es la gamificación, de cómo funciona y especialmente de cómo diseñar experiencias que realmente inspiren cambios de comportamiento o resultados deseables en los jugadores o estudiantes.

Así, la gamificación es una tendencia actual, de uso creciente por parte de los centros de educación y las organizaciones, que pretende llevar a los participantes o usuarios a aprendizajes o comportamientos específicos por medio de elementos del juego, pero tiene grandes retos a superar, que permitan su expansión y uso formal en diferentes disciplinas.

\section{Metodología}

Para esta investigación se realizó una búsqueda bibliográfica sobre los temas relacionados con los juegos en la educación, para lo cual se tomaron los términos: juegos de aprendizaje, aprendizaje basado en juegos, juegos serios y juegos educativos y gamificación, comúnmente utilizados en la literatura para designar las herramientas asociadas a los juegos y diseñadas con objetivos específicos de aprendizaje. Se tomó el período entre 1960 y 2019, de las publicaciones tipo artículo, libro, capítulos de libro y conferencias, en idiomas inglés, español y francés, para lo cual se utilizó como referencia la base de datos Scopus. En la Tabla 1 se muestra el total de documentos recuperados para cada concepto buscado.
Tabla 1. Documentación bibliográfica sobre juegos en la educación, 1960-2019

\begin{tabular}{|l|c|}
\hline \multicolumn{1}{|c|}{ Conceptos } & Documentos \\
\hline Game based learning & 17.430 \\
\hline Serious games & 9.262 \\
\hline Learning games & 34.675 \\
\hline Educational games & 11.341 \\
\hline Gamification & 5.481 \\
\hline
\end{tabular}

Fuente: elaboración propia a partir de la base de datos Scopus.

Posterior a la búsqueda, se realizó una revisión de literatura basada en la guía propuesta por Velásquez (2015), en la cual se utilizaron los términos: educational games, serious games y gamification. Con dicha revisión se busca responder a las siguientes preguntas: ¿cuáles son las características propias de los juegos educativos, juegos serios y la gamificación?, ¿qué características tienen en común estos tres términos?, ¿cómo se relacionan los juegos educativos, los juegos serios y la gamificación?

Para estas búsquedas se definieron criterios de exclusión cómo: áreas de estudio diferentes a ingeniería, ciencias de la computación, ciencias sociales y negocios o administración, casos de estudio o aplicación de los conceptos. El principal criterio de inclusión utilizado fue que se tratara de documentos con amplio marco teórico, revisiones de literatura o que mostraran las características conceptuales de los términos estudiados.

Adicionalmente, se realizó una clasificación de los documentos teniendo en cuenta la variable "cantidad de citas"y se procedió al análisis de los documentos más citados en cada una de las categorías estudiadas, que además tuvieran elementos que respondieran con mayor precisión a las preguntas de investigación planteadas. Para complementar la revisión, se incluyeron libros y documentos con referentes teóricos por fuera de la base de datos usada.

Para el análisis de los documentos, se tomaron las definiciones y características mostradas para cada uno de los términos estudiados. Posteriormente, se 
realizó un comparativo entre las diferentes características determinadas y se tomaron las que se presentaran con mayor frecuencia. Dicho procedimiento se realizó para cada uno de los conceptos estudiados: juegos educativos, juegos serios y gamificación.

\section{Resultados}

Como resultado de las búsquedas bibliográficas, se construyó la Figura 2, en la cual se detalla la cantidad de publicaciones realizadas por año sobre los distintos conceptos: juegos de aprendizaje, aprendizaje basado en juegos, juegos serios y juegos educativos, entre los años 1960 y 2019.

De esta manera, se puede observar que los términos asociados a juegos en la educación han estado presentes en las investigaciones y tienen publicaciones continuas desde 1960, manteniéndose constantes hasta 1990, cuando empiezan a incrementar. Posteriormente, se observa que aproximadamente entre los años 2000 y 2005 hay un incremento importante en el número de publicaciones, hecho que coincide con lo planteado por Djaouti et al. (2011), quienes hacen referencia a una "ola de juegos serios", que pone en el panorama de las personas los juegos como herramientas en la educación. En cuanto a la gamificación, las primeras investigaciones aparecen entre 2011 (22 publicaciones) y 2012 (87 publicaciones), momento a partir del cual incrementan significativamente, llegando a $1137 \mathrm{pu}$ blicaciones durante el 2019.

En cuanto a la procedencia de las publicaciones realizadas en dichos temas, se pudo determinar que la mayor parte de los documentos publicados sobre dichos temas proceden de Estados Unidos (22,5\%), Reino Unido (7,9\%), China (4,9\%), Alemania (4,6\%), Canadá $(3,9 \%)$ y España $(4,1 \%)$, principalmente. Además, en la Figura 3 se muestran las principales áreas del conocimiento en las cuales se han realizado dichas publicaciones. Se puede observar que las ciencias de la computación es el área donde los juegos tienen su mayor impacto, explicado en gran medida por el auge de los videojuegos y de las tecnologías de la información y la comunicación, seguidos por ciencias sociales, ingeniería, matemáticas y medicina.

Figura 2. Documentos publicados por lustro sobre juegos en la educación, 1960-2019

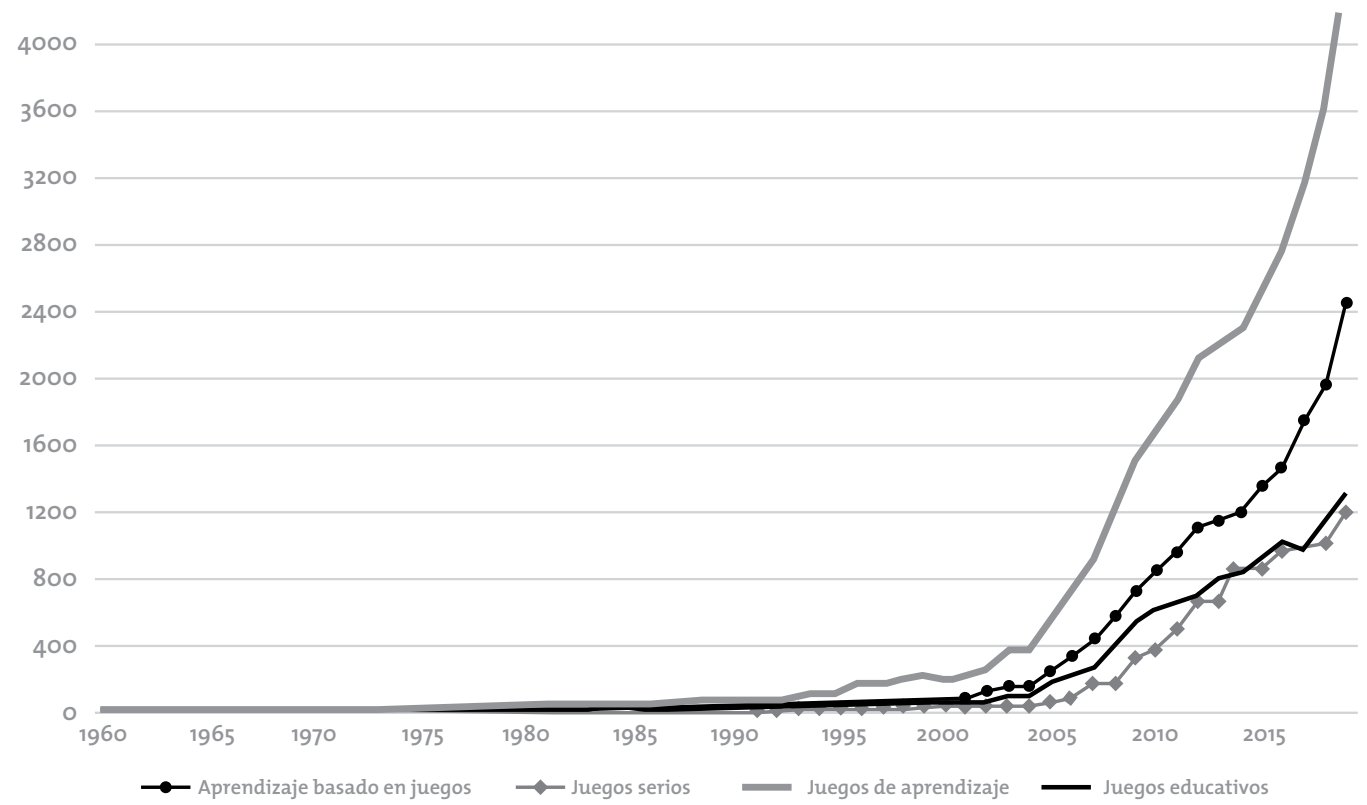

Fuente: elaboración propia a partir de la base de datos Scopus. 


\section{Figura 3. Áreas de aplicación de los juegos para el aprendizaje}

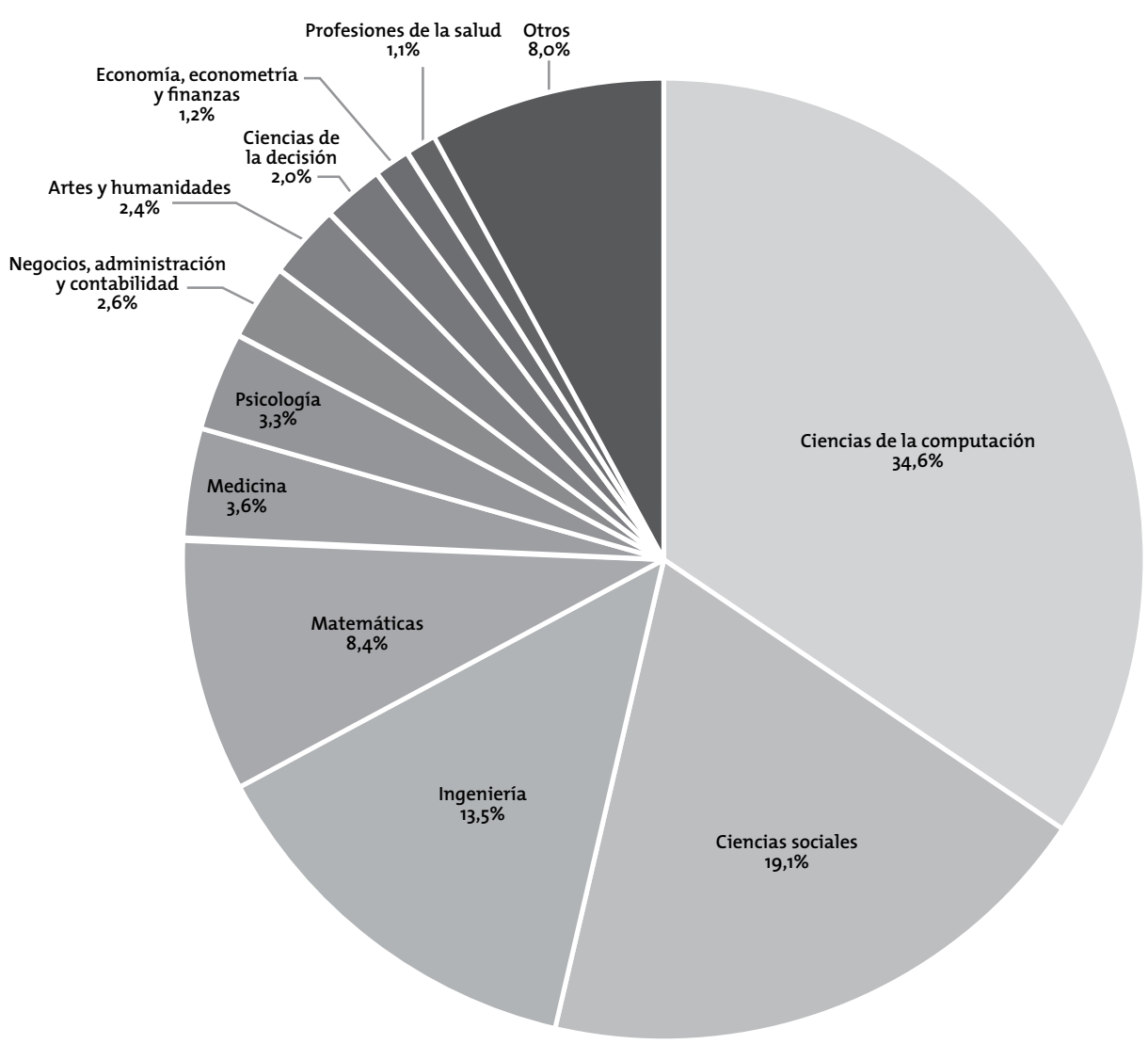

Fuente: elaboración propia a partir de la base de datos Scopus.

Como se puede observar, dentro de los procesos educativos los juegos están presentes en numerosas áreas del conocimiento y las investigaciones relacionadas con estos tienen una tendencia creciente dentro del ámbito académico, lo cual permite el desarrollo de nuevas herramientas y tendencias que se incorporan a las dinámicas propias de los procesos de enseñanza-aprendizaje.

A continuación, con base en la revisión bibliográfica realizada, se pudo determinar la evolución de dichos juegos durante los siglos XX y XXI, como se muestra en la Figura 4. Se inicia con los conceptos básicos de juego que aparece en 1949 con la publicación del libro Homo ludens, pasando por la aparición de los juegos serios y finalizando con la reciente tendencia a la gamificación.
Una vez mostrada la evolución de los juegos hasta lo que hoy se conoce como gamificación, se realizó un análisis comparativo de los conceptos y definiciones de cada uno de los términos estudiados: juegos educativos, juegos serios y gamificación, con el objetivo de encontrar características y elementos propios de cada uno de estos.

Uno de los principales hallazgos con respecto a los juegos en la educación es que se hace necesario diferenciar play de game, dos términos que en español son usualmente utilizados de manera indistinta, pero que hacen referencia a actividades diferentes e igualmente tienen características diferenciadas. En la Tabla 2 se muestran las principales características determinadas para cada uno de los conceptos estudiados. 
Figura 4. Evolución de los juegos en contextos educativos

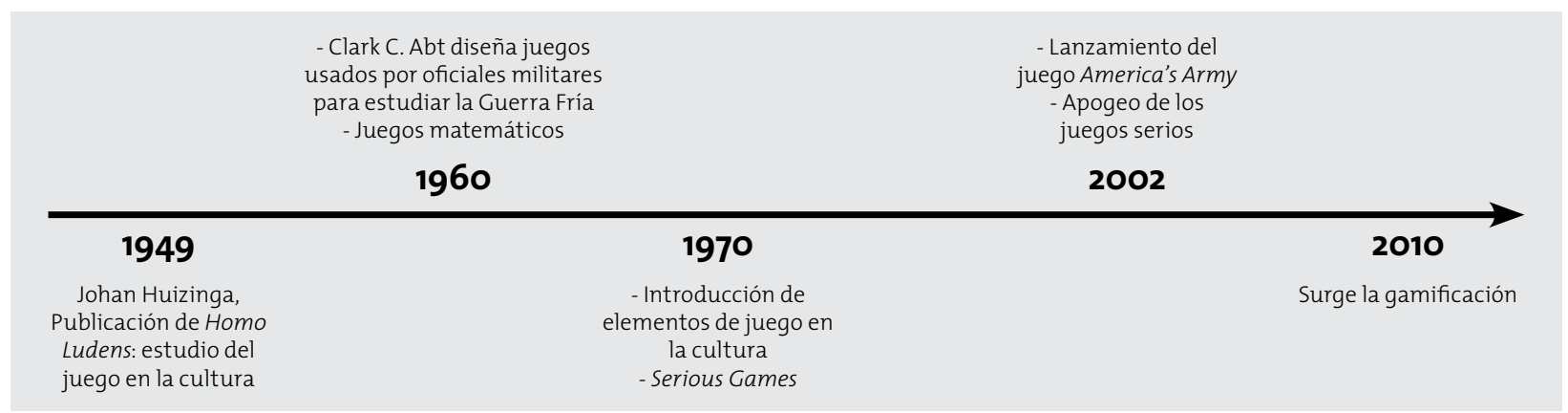

Fuente: elaboración propia.

Tabla 2. Principales caracteristicas de los juegos educativos, juegos serios y gamificación

\begin{tabular}{|c|l|l|}
\hline \multirow{2}{*}{ Concepto } & \multicolumn{1}{|c|}{ Caracteristicas } \\
\hline \multirow{2}{*}{ Juegos } & \multicolumn{1}{|c|}{ Play } & \multicolumn{1}{c|}{ Game } \\
\cline { 2 - 3 } & $\begin{array}{l}\text { Juegos libres } \\
\text { Actividades lúdicas } \\
\text { Sin objetivos determinados } \\
\text { Surgen de la propia motivación }\end{array}$ & $\begin{array}{l}\text { Juegos con normas específicas } \\
\text { Sometido a reglas, límites u obligaciones } \\
\text { Espacio de juego con límites y contexto determinado } \\
\text { Mundo que evoluciona en etapas } \\
\text { Objetivos concretos } \\
\text { Resultados y retroalimentación }\end{array}$ \\
\hline \multirow{5}{*}{ Juegos serios } & $\begin{array}{l}\text { Juegos enfocados en la enseñanza } \\
\text { Pueden o no ser herramientas computarizadas } \\
\text { Sistema de reglas y meta clara } \\
\text { Modelar situaciones reales } \\
\text { Interfaz fácil y amigable con el usuario }\end{array}$ \\
\hline \multirow{6}{*}{ Gamificación } & $\begin{array}{l}\text { Técnica reciente que utiliza elementos propios de los juegos } \\
\text { Puntos, insignias, niveles, rankings } \\
\text { Tiene objetivos claros y concretos } \\
\text { Contexto determinado y límites } \\
\text { Utilizado en contextos que no son juegos } \\
\text { Revela el potencial creativo }\end{array}$ \\
\hline
\end{tabular}

Fuente: elaboración propia.

Adicional a las características mostradas, se encontraron tres variables presentes en los análisis realizados: 1) aprendizaje: dado que los tres conceptos hacen referencia a herramientas utilizadas en la enseñanza, pero que no funcionan o enseñan de la misma manera; 2) diversión: elemento no necesariamente presente en todos los conceptos, pero que juega un papel relevante en el diseño de las herramientas y en la motivación de los participantes; $\mathrm{y}$ 3) resultados: se pueden obtener con la aplicación de estas herramientas o los objetivos que se persiguen.
Adicionalmente, se incluyó el elemento uso, que hace referencia a los grupos de personas, centros de educación o procesos en los cuales cada herramienta es utilizada en mayor medida.

Las características comunes identificadas en cada análisis comparativo, y los elementos diversión, aprendizaje, resultados y uso de cada uno de los conceptos estudiados fueron el insumo con el cual se construyó una matriz general (Tabla 3), que resume la información. 
Tabla 3. Matriz de caracteristicas asociadas de los juegos, juegos serios y gamificación

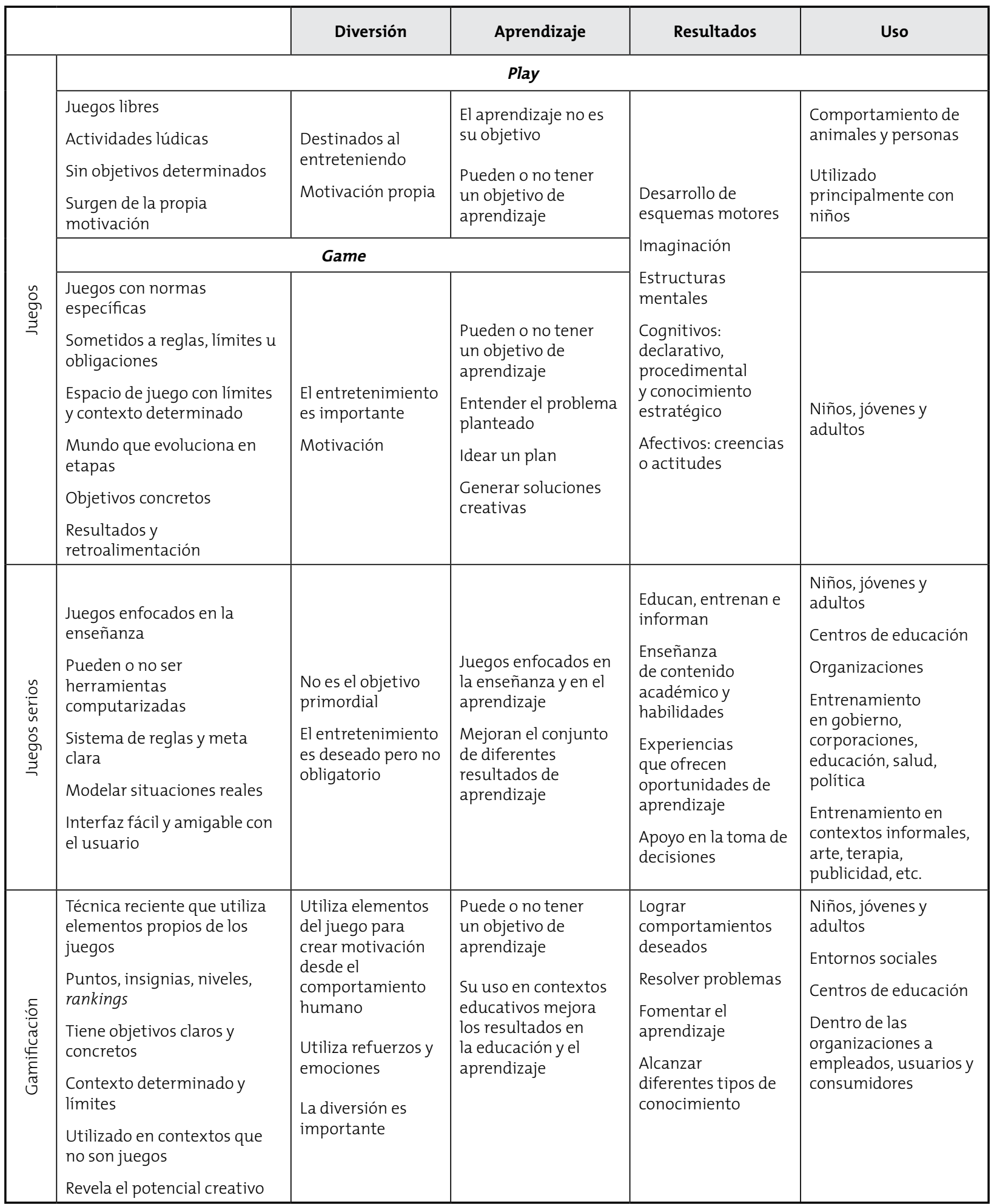

Fuente: elaboración propia. 
A partir de la información consignada en la matriz, se desarrolló un modelo teórico integrador de los conceptos asociados a los juegos en la educación estudiados, el cual intenta mostrar gráficamente las relaciones existentes entre estos, a partir de los elementos determinados anteriormente: características, diversión, aprendizaje y resultados (Figura 5).

Para el desarrollo del modelo teórico se tomaron los conceptos de juego, juegos serios y gamificación, como bloques principales independientes, pero relacionados entre sí. La línea punteada hace referencia al concepto de aprendizaje basado en juegos, es decir, el aprendizaje que se obtiene por medio de la aplicación de juegos dentro de los procesos de enseñanza; dentro de este conjunto prevalece el aprendizaje y por fuera de él prevalece la diversión; sin embargo, siempre hay un intercambio constante entre dichas nociones.
El primer bloque hace referencia al concepto general de juego, el cual se subdivide en dos partes: la primera enuncia los juegos libres, mientras que la segunda enuncia los estructurados. Sus resultados pueden o no constituir aprendizaje, dado que dichos juegos pueden aplicarse únicamente con un objetivo de entretenimiento, razón por la cual parte de dicho bloque se encuentra por fuera del conjunto de aprendizaje basado en juegos.

El segundo bloque hace referencia a los juegos serios, los cuales tienen por objetivo principal el aprendizaje. En estos, la diversión no es la finalidad; sin embargo, es deseable y existe un esfuerzo constante para que dicho elemento esté presente en las herramientas propuestas. Este bloque se encuentra completamente dentro del aprendizaje basado en juegos, dado que siempre hay un objetivo de aprendizaje.

Figura 5. Modelo teórico integrador de los conceptos asociados a los juegos en la educación

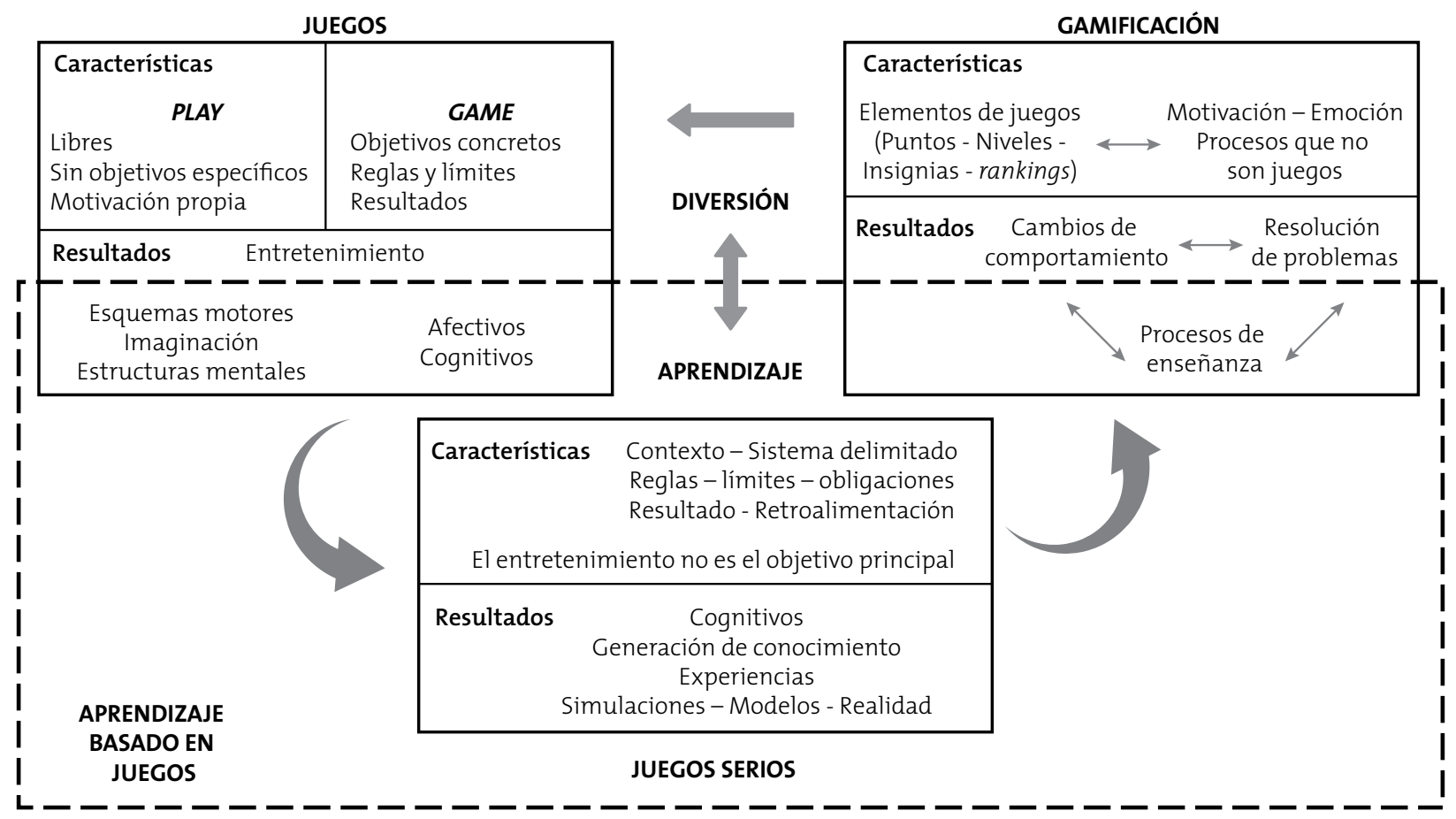

Fuente: elaboración propia. 
Finalmente, el tercer bloque se centra en la gamificación, donde la diversión y la motivación son fundamentales para el logro de los objetivos. Al igual que el concepto general de juego, esta puede o no tener resultados de aprendizaje, debido a que también es utilizada para lograr cambios de comportamientos y resolución de problemas, no necesariamente resultado de un proceso de aprendizaje. Sin embargo, dichos resultados se relacionan entre sí, dependiendo de las herramientas desarrolladas.

\section{Conclusiones}

Como se observó, los juegos son actividades que están presentes desde la infancia en las personas, como acciones que promueven el desarrollo sicológico y motriz de niños, pero que no son exclusivos de estas edades, dado su creciente desarrollo en diversas áreas del conocimiento y su aplicación en todos los niveles educativos y organizacionales, para lograr un aprendizaje efectivo.

En años recientes, estos se han convertido un área relevante para colegios, universidades, organizaciones y comunidad general, debido al impacto en procesos de aprendizaje y entrenamiento. Adicionalmente, constituyen un amplio campo de estudio, definido formalmente en el siglo XX, y desde entonces han logrado experiencias significativas en diseño y aplicación de juegos. Como se pudo observar en el documento, tanto los juegos en la educación y como los conceptos que han surgido a partir del mismo son objeto de numerosas investigaciones académicas y organizacionales, que los han convertido en un tema de interés actual para los centros de educación a nivel mundial.

Los juegos serios y la gamificación son herramientas que permiten a los participantes aprender haciendo, mediante el uso de tecnologías y elementos que logran ambientes de aprendizaje amigables y motivantes, que incluso pueden trascender la educación e impactar áreas organizacionales y sociales.

De esta manera, el modelo propuesto logra integrar varios de los conceptos relacionados con juegos en la educación y mostrar sus características y resultados principales, a partir de la relación entre diversión y aprendizaje. Ello permite a la vez clarificar, explicar y asociar los elementos presentes en cada uno de dichos conceptos.

No obstante, los juegos son un campo de conocimiento en construcción y relativamente nuevo, aunque se desarrolla con agilidad, y tiene alta relevancia en los modelos de educación y aprendizaje. Existen varios desafíos para los investigadores en el tema, como son: la aplicación de herramientas de evaluación eficaces, el análisis de datos de comportamiento resultado de aplicación de juegos, el balance entre aprendizaje y diversión, la manera de plasmar la realidad y verdaderas necesidades académicas de los participantes, la falta de metodologías y modelos unificados, entre otros interrogantes que deberán ser abordados por los investigadores del tema en los próximos años.

\section{Referencias}

Abt, C. C. (1970). Serious games. University Press of America.

Ahrens, D. (2015). Serious games - A new perspective on workbased learning. Procedia - Social and Behavioral Sciences, 204 (agosto), 277-281. https://doi.org/10.1016/j.sbspro.2015.08.152

Arnab, S., Berta, R., Earp, J., De Freitas, S. y Popescu, M. (2012). Framing the adoption of serious games in formal education. EJEL. e-Journal of e-Learning, 10(2), 159-171. http://www.ejel.org/volume10/issue2 
Ben-Zvi, T. (2010). The efficacy of business simulation games in creating Decision Support Systems: An experimental investigation. Decision Support Systems, 49(1), 61-69. https://doi.org/10.1016/j.dss.2010.01.002

Bernabeu, N. y Goldstein, A. (2012). Creatividad y aprendizaje. El juego como herramienta pedagógica. Narcea.

Boyle, E. A., Connolly, T. M. y Hainey, T. (2011). The role of psychology in understanding the impact of computer games. Entertainment Computing, 2, 69-74.

Boyle, E. A., Hainey, T., Connolly, T. M., Gray, G., Earp, J., Ott, M., ... Pereira, J. (2016). An update to the systematic literature review of empirical evidence of the impacts and outcomes of computer games and serious games. Computers \& Education, 94, 178-192. https://doi.org/10.1016/j.compedu.2015.11.003

Braghirolli, L. F., Ribeiro, J. L. D., Weise, A. D. y Pizzolato, M. (2016). Benefits of educational games as an introductory activity in industrial engineering education. Computers in Human Behavior, 58, 315-324. https://doi. org/10.1016/j.chb.2015.12.063

Breuer, J. y Bente, G. (2010). Why so serious? On the relation of serious games and learning. Eludamos. Journal for Computer Game Culture, 4(1), 7-24. http://www.eludamos.org/index.php/eludamos/article/view/vol4no1-2

Connolly, T. M., Boyle, E. A., MacArthur, E., Hainey, T. y Boyle, J. M. (2012). A systematic literature review of empirical evidence on computer games and serious games. Computers \& Education, 59(2), 661-686. https://doi. org/10.1016/j.compedu.2012.03.004

Deterding, S., Dixon, D., Khaled, R.y Nacke, L. (2011). From game design elements to gamefulness: Defining "gamification". En MindTrek '11: Proceedings of the 15 th International Academic MindTrek Conference: Envisioning Future Media Environments, Tampere Finland.

Deterding, S., Dixon, D., Khaled, R.y Nacke, L. (2011). Gamification:Toward a definition. CHI (mayo 7-12). Vancouver, Canada.

Djaouti, D., Álvarez, J., Jessel, J.-P. y Rampnoux, O. (2011). Origins of serious games. En Serious games and edutainment applications (pp. 25-43). Springer.

Emel'yanenko, V. D., Vetoshko, A. N., Malinnikov, S. G., Malashenko, I. V. y Vetoshko, L. I. (2016). Man's values and ideologies as a basis of gamification. International Journal of Environmental and Science Education, 11(18), 12576-12592. https://files.eric.ed.gov/fulltext/EJ1124629.pdf

Ferrara, J. (2013). Games for persuasion: Argumentation, procedurality, and the lie of gamification. Games and Culture, 8(4), 289-304. https://doi.org/10.1177/1555412013496891

Gray, D., Brown, S. y Macanufo, J. (2012). Gamestorming: 83 juegos para innovadores, inconformistas y generadores del cambio. Deusto.

Groh, F. (2012). Gamification: State of the art definition and utilization. Conference: Proceedings of the $4^{\text {th }}$ Seminar on Research Trends in Media Informatics (pp. 39-46). RTMI. 
Hamari, J., Koivisto, J. y Sarsa, H. (2014). Does gamification work? - A literature review of empirical studies on gamification. Proceedings of the Annual Hawaii International Conference on System Sciences, Waikoloa, HI, EE.UU. (pp. 3025-3034). IEEE. https://doi.org/10.1109/HICSS.2014.377

Huizinga, J. (1949). Homo ludens: A study of the play element in culture. Routledge \& Kegan Paul. https://eva.udelar.edu.uy/pluginfile.php/1074698/mod_resource/content/3/Huizinga\%2O-\%2OHomo\%2OLudens\%2O \%281\%29.pdf

Huotari, K. y Hamari, J. (2011). "Gamification” from the perspective of service marketing. CHI (mayo 7-12). http:// gamification-research.org/wp-content/uploads/2011/04/14-Huotari.pdf

Iten, N. y Petko, D. (2016). Learning with serious games: Is fun playing the game a predictor of learning success? British Journal of Educational Technology, 47(1), 151-163. https://doi.org/10.1111/bjet.12226

Juul, J. (2010). The game, the player, the world: Looking for a heart of gameness. Plurais - Revista Multidisciplinar, 1(2). https://revistas.uneb.br/index.php/plurais/article/view/880

Kafai, Y. B.y Burke, Q. (2015). Constructionist gaming: Understanding the benefits of making games for learning. Educational Psychologist, 50(4), 313-334. https://doi.org/10.1080/00461520.2015.1124022

Kapp, K. M. (2012). The gamification of learning and instruction: game-based methods and strategies for training and education. Pfeiffer.

Khenissi, M. A., Essalmi, F.y Jemni, M. (2015). Comparison between serious games and learning version of existing games. Procedia - Social and Behavioral Sciences, 191, 487-494. https://doi.org/10.1016/j.sbspro.2015.04.380

Landers, R. N. (2014). Developing a theory of gamified learning: Linking serious games and gamification of learning. Simulation \& Gaming, 45(6), 752-768. https://doi.org/10.1177/1046878114563660

Lauche, K., Crichton, M. y Bayerl, P. S. (2009). Tactical decision games: Developing scenario-based training for decision-making in distributed teams. Proceedings of the NDMg, $9^{\text {th }}$ International Conference on Naturalistic Decision Making, Londres.

Lee, J. y Hammer, J. (2011). Gamification in education: What, how, why bother? Academic Exchange Quarterly, 15(2). https://www.uwstout.edu/soe/profdev/resources/upload/Lee-Hammer-AEQ-2011.pdf

Liu, C.-C., Cheng, Y.-B. y Huang, C.-W. (2011). The effect of simulation games on the learning of computational problem solving. Computers \& Education, 57(3),1907-1918. https://doi.org/10.1016/j.compedu.2011.04.002

Londoño, L. M. (2014). Diseño de un juego para enseñar y reforzar las relaciones de confianza. Universidad Nacional de Colombia.

Medrano, N. (2005). El gran libro de los juegos de mesa. Andrómeda.

Michael, D. R.y Chen, S. (2006). Serious games: Games that educate, train, and inform. Thomson Course Technology. 
Moreno-Ger, P., Burgos, D., Martínez-Ortiz, I., Sierra, J. L. y Fernández-Manjón, B. (2008). Educational game design for online education. Computers in Human Behavior, 24(6), 2530-2540. https://doi.org/10.1016/j. chb.2008.03.012

Ouariachi, T., Gutiérrez-Pérez, J.y Olvera-Lobo, M.-D. (2017). Criterios de evaluación de juegos en línea sobre cambio climático. Revista Mexicana de Investigación Educativa, 22(73), 445-474. http://www.scielo.org.mx/scielo.php?script=sci_abstract\&pid=S1405-66662017000200445

Riemer, V.y Schrader, C. (2015). Learning with quizzes, simulations, and adventures: Students' attitudes, perceptions and intentions to learn with different types of serious games. Computers \& Education, 88, 160-168. https://doi.org/10.1016/j.compedu.2015.05.003

Robson, K., Plangger, K., Kietzmann, J. H., McCarthy, I.y Pitt, L. (2015). Is it all a game? Understanding the principles of gamification. Business Horizons, 58(4), 411-420. https://doi.org/10.1016/j.bushor.2015.03.006

Salen, K. y Zimmerman, E. (2004). Rules of play: Game Design fundamentals. MIT Press.

Sauvé, L., Renaud, L.y Gauvin, M. (2009). Une analyse des écrits sur les impacts du jeu sur l'apprentissage. Revue des Sciences de L'éducation, 33(1), 89-107. https://doi.org/10.7202/01619oar

Sawyer, B. (2007). The "Serious Games" landscape. The Instructional \& Research Technology Symposium for Arts, Humanities and Social Sciences, Camden, EE.UU. https://doi.org/10.1007/978-1-4471-2161-9_3

Smith-Robbins, S. (2011). "This game sucks”: How to improve the gamification of higher education. New Horizons.

Teixes, F. (2014). Gamificación: Fundamentos y aplicaciones. UOC.

Velásquez, J. D. (2015). Una guía corta para escribir revisiones sistemáticas de literatura Parte 4. DYNA, 82(190), 9-12. https://doi.org/10.15446/dyna.v82n190.49511

Waiyakoon, S., Khlaisang, J.y Koraneekij, P. (2015). Development of an instructional learning object design model for tablets using game-based learning with scaffolding to enhance mathematical concepts for mathematic learning disability students. Procedia - Social and Behavioral Sciences, 174, 1489-1496. https://doi. org/10.1016/j.sbspro.2015.01.779

Zyda, M. (2005). From visual simulation to virtual reality to games. Computer, 38(9), 25-32. http://mikezyda. com/resources/pubs/Zyda-IEEE-Computer-Sept2005.pdf 